Document downloaded from:

http://hdl.handle.net/10251/180614

This paper must be cited as:

Sendra, S.; García-Navas, JL.; Romero-Diaz, P.; Lloret, J. (2019). Collaborative LoRaBased Sensor Network for Pollution Monitoring in Smart Cities. IEEE. 318-323. https://doi.org/10.1109/FMEC.2019.8795321

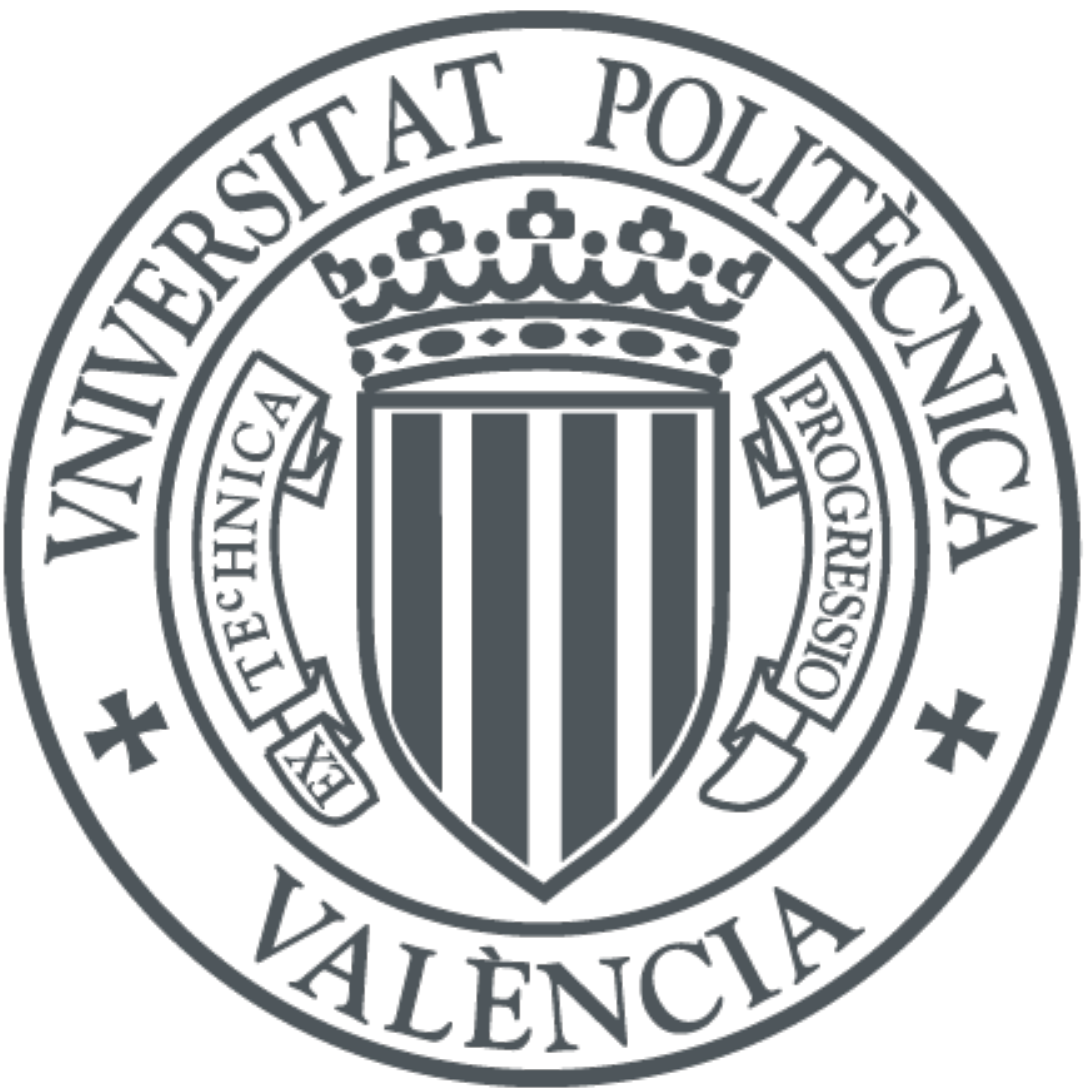

The final publication is available at

https://doi.org/10.1109/FMEC.2019.8795321

Copyright IEEE

Additional Information 


\title{
Collaborative LoRa-Based Sensor Network for Pollution Monitoring in Smart Cities
}

\author{
Sandra Sendra ${ }^{1,2}$, Jose Luis Garcia-Navas ${ }^{2}$, Pablo Romero-Diaz ${ }^{1}$, Jaime Lloret ${ }^{2}$ \\ ${ }^{l}$ Deptarmento de teoría de la señal, telemática y comunicaciones \\ Uniersidad de Granada \\ Granada, Spain \\ ${ }^{2}$ Intituto de Inestigacion para la Gestion Integrada de zonas Costeras \\ Universitat Politècnica de València \\ Valencia, Spain \\ ssendra@ugr.es,jogarna3@teleco.upv.es, pabloromerodiaz@ugr.es, jlloret@dcom.upv.es
}

\begin{abstract}
With the aging of the car park in big cities, together with the fact that the number of users of private vehicles is increasing, the quality of air (QoA) in large cities has been worryingly reduced. Therefore, it is becoming increasingly necessary to implement new monitoring infrastructures to improve our smart cities. Therefore, in this paper, we present a collaborative Long Range (LoRa)-based sensor network for pollution monitoring in smart cities. The system is based on geo-located nodes capable of measuring the temperature, relative humidity and concentration of $\mathrm{CO} 2$ in urban environments. The system also takes into account the subjective opinions of citizens. By using the collected data and considering the restrictions policies of the city, the system in able to send the required orders to panels and traffic signs to control de traffic circulation. Finally, the citizens can be advised to avoid going to too polluted areas. The system has been testes in a real environment with very interesting results.
\end{abstract}

Keywords-collaborative network, smart city, pollution, sensors, wireless sensor network (WSN), Long Range (LoRa), Internet of Things (IoT).

\section{INTRODUCTION}

The increasing trend in population migration into urban center and the exponential growing of Information and communication technologies (ICT), has led to the appearance of the concept of Smart City that has six main dimensions: smart economy, smart people, smart governance, smart mobility, smart environment, and smart living [1]. By 2050, $70 \%$ of the world population will live in the urban center [2]. Looking back, Internet of Things (IoT's) roots can be traced to 1999 when Auto-ID center was founded in Massachusetts Institute of Technology (MIT). In 2003 there were approximately 6.3 billion people living on the planet and 500 million devices connected to the Internet, 0.08 devices per person. In 2010, the number of devices connected to the Internet brought to 12.5 billion, 1.84 devices device for every person (6.8 billion people in the world). Looking to the future, Cisco IBSG predicts there will be 50 billion by 2020 , what means 6.58 devices per person ( 7.6 billion people in the world) [3]. From the point of view of the technology, Smart Cities are thought to improve the lifestyle of people, and its growth is undoubted.

Cities can influence health and disease in many ways. Urban living often coincides with risk factors as exposures. One of the most important is ambient air pollution. Worldwide, air pollution varies strongly by region. In increasing order of ambient air pollution, North America and Australia are the less polluted regions, followed by central Europe and finally India and Asia, with the highest ambient air pollution. For South America and Africa there is few information, but the information available shows high air pollution levels [4]. The increasing of the population of the urban cities and the fast growing of industrial activities increases the problem of air pollution and justify the necessity of monitoring it. Monitor air pollution allows controlling the Quality of Air (QoA) [5].

Thinking on the size of a city and how to monitor factors such as air pollution, a good solution could consist of an infrastructure composed of sensors connected wirelessly. That could permit centralize all the sensors information, control it and used it to take advantage of. Wireless technologies allow a large deployment by low cost, but it all depends on the technology selected. The principal disadvantage is the differences between all the wireless technologies of connectivity and how to combine them in order to satisfy the requirements of the communications [6]. Wireless sensor networks are increasingly being deployed in areas where several types of information need to be harvested. These networks need to guarantee a maximal coverage of the Area of Interest and then establish an algorithm that minimizes energy consumption [7]. Permanent connectivity is also required in most of the wireless networks, what suppose a challenge because of several technical weak points such us low battery, connectivity range, monitoring range and difficulties in deployment [7].

As far as wireless connectivity is concerned, there are two developed scenarios: nearby areas that could be around hundreds of meters where technologies such as Bluetooth, WiFi or Zigbee can be used; and a remote area where cabling is not an option and cellular mobile network modems, or satellite modems are needed. Between those scenarios there are some situations that cost-benefit is insufficient to justify mobile network connection and the distances to cover are too great to use nearby technologies. This gives sense to the emergence of technologies such as LoRa (Long-Range) [8]. LoRa technology operates on the 433,868 or $915 \mathrm{MHz}$ ISM bands. Their uses depend on the region in which it is deployed [9] and it is based on a proprietary spread spectrum technique and Gaussian Frequency Shift Keying. LoRa includes two major components. The first is modulation based on Chirp Spread Spectrum (CSS), LoRa uses wideband linear frequency modulated pulse whose frequency depends on the encoded information. That makes the signal resistant against interferences. Also, LoRa modulation features in six orthogonal spreading factors (SF) resulting in the different data rates, in order to improve spectral efficiency and increase the capacity of the network. And the 
second component is a MAC layer protocol (LoRaWAN) which is optimized specifically for energy limited End Devices [10].

As a summary, the concept of Smart City covers a wide scope of applications to improve lifestyle. One of the most important factor for the development of Smart Cities, and in this case related to the air pollution monitoring, is the correct interconnection and architecture of the different infrastructures. In that way, one of the most interesting architectural proposals is the group-based networks [11]. Group-based networks applied in Smart City networks will improve the performance and the efficiency of the network, permit a more flexible and efficient sensors operation and will imply low energy consumption that suppose an increase of the network lifetime. Group-based networks are based on the idea of dividing wireless sensors into multiple groups and monitor these groups, establishing required connectivity between these groups [12]. Group-based networks try to offer the same or better services of typical network reducing the cost.

Taking into account the aforementioned issues, this paper presents a collaborative LoRa-based sensor network for pollution monitoring in smart cities. The system is based on the use of LoRa-based nodes which incorporates a GPS interface. The nodes are installed in both vehicles and fixed points in the city. The LoRa-based nodes are in charge of collecting data of temperature, relative humidity and $\mathrm{CO}_{2}$ concentrations. These data is also combined with the opinion of citizens. Finally, the collected data is used to autonomously generate (considering the local restrictions policies) the orders to be sent to traffic signs and panels and the citizens receive some messages with recommendations. The system has been teste in an actual scenario.

The remainder of this paper is organized as follows. Section II presents some related work. Fundamentals of our proposal and the hardware used to implement our nodes are explained in Section III. Section IV depicts the collaborative algoritmh used to collect the data and generate the warnings . Section V describes the performance evaluation experiments and results. Finally, Section VI draws the main conclusions and future work.

\section{RELATED WORK}

This section collects the most important wireless sensor networks implementations for pollution monitoring. We have classified them by the wireless technology used by the wireless sensor network.

M. F. Othman et al. [13] discuss and review the application study of WSNs in environmental monitoring as an alternative way of conventional method. These approaches can improve the system performance, provide a convenient and efficient method, and can also fulfill functional requirements. Wireless Sensor Network (WSN) have increased the growth of low cost, low power and multifunctional sensors that are small in size and can process data, execute tasks, assist other nodes and transmit the processed data to sink node over a wireless communication. Each node consists of micro-controllers, memory and transceiver. Nodes are used to monitor and control the physical environment from remote locations. Finally, Sink node collects data and transmits the analyzed data to user via Internet.
W.Y. Yi et al. [14] perform a classification of three different existing technologies: Static Sensor Network (SSN), Community Sensor Network (CSN) and Vehicle Sensor Network (VSN). They discuss the limitations of them and conclude the objectives to achieve in future systems, in order to monitor air pollution. Researchers are pushing the concept of The Next Generation Air Pollution Monitoring System (TNGAPMS) and have achieved great progresses, by using WSN and Micro-Electro-Mechanical Systems. Air pollution monitoring systems evidence that having systems with high spatio-temporal resolution, cost and energy efficiency, deployment and maintenance visibility are achievable. However, some abilities or characteristics of these existing systems needs to carry forward or enhance when building the future systems, in order to reduced system and maintenance cost and to improve the data quality and cost efficiency.

A. Kadri et al. propose [15] an ambient real-time air quality monitoring system, that consists of several distributed monitoring multi-gas (MG) stations that communicate wirelessly with a backend server using machine-to-machine (M2M) communication. Each station is equipped with gaseous and meteorological sensors as well as data logging. The backend server collects real time data from the stations, processes and delivers it to users through web portals and mobile applications. They describe a deployed and functional comprehensive end-to-end system. In addition, the system uses a high density of sensing stations per unit area in order to provide localized pollution information. Additional techniques under current investigation include the use of prediction algorithms based on neural networks in order to estimate pollution information in the near future.

In [16], A. Mehmood et al. present a novel Secure and Low-energy Zone-based Routing Protocol (SeLeZoR) for pollution monitoring. Nodes of the WSN are split into zones. Each zone is separated into clusters, each one controlled by a cluster head $(\mathrm{CH})$. SeLeZoR implements several improvements to enhance the issue of energy consumption, reducing the needless waste of energy, and data vulnerability. The data are exchanged between intra-zone communication and inter-zone communication using a secret key and a secure and energy efficient mechanism, in order to identify the malicious data while maintaining the network performance. In SeLeZoR, zone-based technique reduces communication messages with the BS and increases the network lifetime. The clusters nearer to the BS have smaller sizes than those clusters away from the BS. SeLeZor has proposed the Vice Head mechanism for $\mathrm{ZH}$ and $\mathrm{CH}$ recovery to avoid the Heads from being a single point of failure.

K. K. Khedo et al. [5] propose the use of an innovative system named Wireless Sensor Network Air Pollution Monitoring System (WAPMS) to monitor air pollution using wireless sensors deployed in huge numbers around the island Mauritius. That system uses an Air Quality Index (AQI) to categorize levels of air pollution, associating different colors to each level, in order to communicate it easily. They designed and implemented a new data aggregation algorithm named Recursive Converging Quartiles (RCQ) that merge data, eliminate duplicates, filter out invalid readings and summarize them into a simpler form that reduces the amount of data and save energy. The protocol uses a hierarchical routing protocol in WAPMS and sleeping times, for better power management. WAPMS will provide real-time 
information and provide alerts in cases of drastic change in quality of air. WAPMS is very flexible, very easy and yet very powerful due to its ability to provide highly summarized results.

Finally, A. Mehmood et al. [17] propose an artificial neural network, based energy-efficient, capable to increase sensor nodes' lifetime, and robust routing scheme for WSNs called ELDC. This protocol extends a previous protocol (EEMDC) by adding some advantageous features of both EEUC and group-based energy saving technique. In this Protocol, the network is trained on huge data set containing almost all scenarios to make the network more reliable and adaptive to the environment. Additionally, it uses groupbased methodology to increase the life-span of the overall network, where groups may have different sizes. Network has an intelligent, efficient, and robust group organization based on back propagation technique, controlled by a group's chief node and a cluster head. It uses Artificial Neural Network for training the protocol by taking into account residual energy, distance of node from $\mathrm{CH}$, distance of $\mathrm{CH}$ from Chief node or boarder node, distance of the boarder node to the next Chief node or the BS, traffic load on the particular link, and the probability of health status of the particular path. Simulation results show that it outperforms LEACH protocol by $42 \%$, and other state-of-the- art protocols by more than $30 \%$. If some nodes consume their energy quickly, then the other nodes support them and let them be in sleep mode.

As far as we know, there are fixed infrastructures to measure air quality in cities but no collaborative technique is applied to enhance the results estimations and to generate autonomous decisions to apply corrective actions and restictions for the traffic circulation. Our system, in addition to measure the environmental parameters, takes into account the citizen opinion regarding to QoA to finally, generate these autonomous decisions to apply corrective actions with the goal of face the problems of pollution in cities.

\section{PROPOSAL DESCRIPTION}

This section presents our proposal. It includes the description of the smart city architecture and the different elements that form part of this architecture. The section also presents the hardware elements used to implement our wireless node.

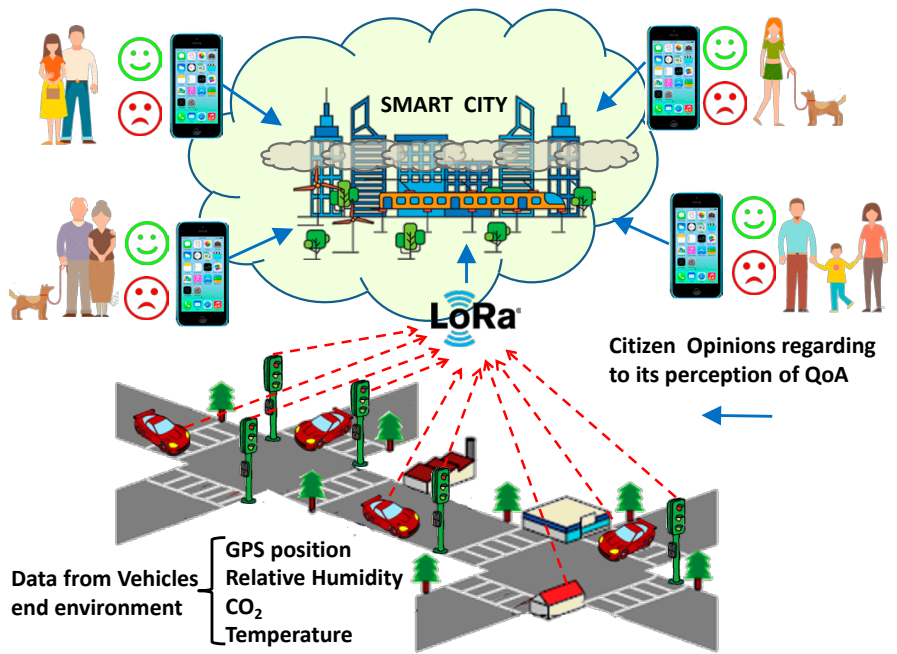

Fig. 1. Collaborative LoRa-Based Sensor Network for Pollution Monitoring in Smart Cities

\section{A. Collaborative smart city framework}

As Figure 1 shows, a smart city can be considered as a collaborative entity [18] capable of combining data from different sources in order to make decisions and take actions to improve a situation.

In our case, we focus our proposal on pollution monitoring in cities. To do this, we consider mobile nodes and fixed nodes, able to take data from the environment continuously. In particular, the installation of wireless motes based on LoRa in vehicles and the installation of motes in fixed elements such as traffic lights and lampposts are considered. With this, we can monitor the evolution of parameters such as temperature, relative humidity and $\mathrm{CO}_{2}$ concentrations in the established points and combine all these data to build real-time maps of the evolution of these parameters. Finally, the proposed network becomes collaborative since the citizen's opinion is considered through a mobile application. These opinions are based on a subjective feedback on the perception of users. With all this information, the system will be able to generate intelligent and autonomous decisions that will be sent to luminous panels and traffic signals in order to control traffic and apply restrictions, if necessary. In addition, the system will generate warning messages to registered users with recommendations on the limitation of transit through certain areas. Figure 2 shows the smart city framework proposed to our collaborative LoRa-based sensor network for pollution monitoring.

\section{B. Node description and sensors used}

To implement each node, we use a TTGO T-Beam node. It is built around the ESP32 chip. It has 4MB of SPI flash. It operates at $433 \mathrm{MHz}, 868 \mathrm{MHz}$ and $915 \mathrm{MHz}$, in our case we use the European ISM band at $868 \mathrm{MHz}$. Our TTGO TBeam node includes two antennas. A GPS ceramic antenna connected to a u-bloc NEO-6M GPS module and another LoRa antenna with SMA connector. It uses a LoRa chip from the HopeRF RFM9X family. The node has a total of 26 pins with GPIO, ADC, VP/VN, DAC, Touch, SPI, I2C, UART and Lora. Finally, the node can be feed by batteries.

In order to measure the temperature and relative humidity, we use a DHT11 sensor. This device offers a digital signal processed through any microprocessor.

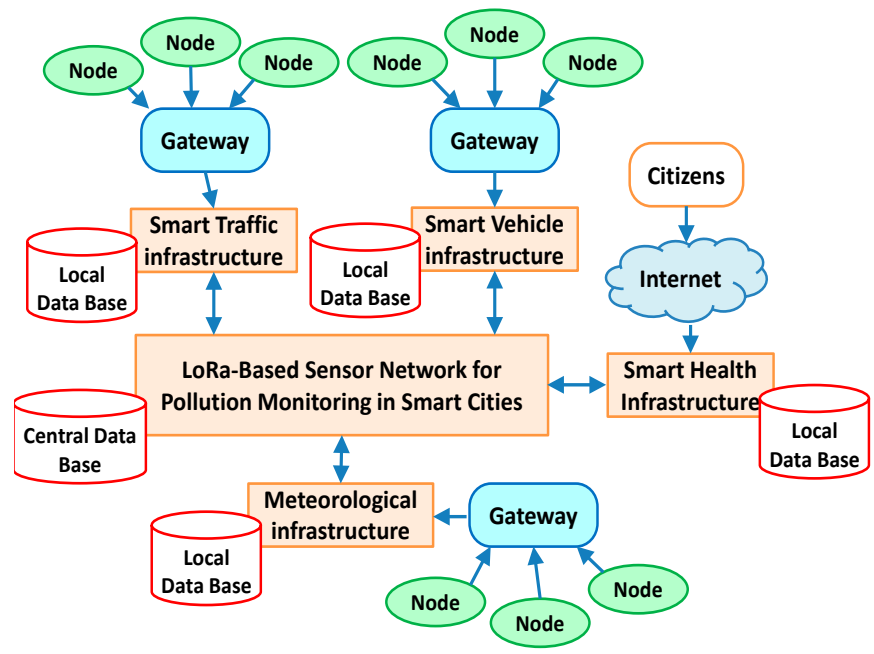

Fig. 2. Smart City framework 
The sensor is connected to a digital pin; however, it works as an analog device. The analog to digital conversion is carried out by the sensor itself. The DHT11 is a small size device with low power consumption very recommended to applications that do not require acquiring data with very low period. The DHT11 operates with operation cycles of $1 \mathrm{~s}$. The DHT11 uses a polymer resistance as a detection element and its operating ranges of this sensor are $20-90 \%$ (with a precision of $\pm 4 \%$ for relative humitidy) and $0-50{ }^{\circ} \mathrm{C}$ (with a precision of $\pm 2{ }^{\circ} \mathrm{C}$ for temperature).

Finally, the measuring of quality of air (QoA) is performed by a MQ-135 sensor. It is an air quality control sensor is used for detecting pollution in the environment. It is usually implemented in control circuits such as alarms in homes and places where it is desired to prevent high levels of pollution that can be harmful to health. This sensor is responsible for the detection of gas concentration in various percentages. The output signal provided by the MQ-135 is dual, i.e., with analog and digital features. Regarding the analog signal provided, this is directly proportional to the voltage increase. The digital signal presents TTL levels so this signal can be easily processed by a microcontroller. The power consumption of the MQ-135 sensor is $800 \mathrm{~mW}$ (Power supply of $5 \mathrm{~V}$ ). It requires a preheating time of 20 seconds and it is able to detect concentrations from $10 \mathrm{ppm}$ to $1000 \mathrm{ppm}$. Its operating temperature is between $-20^{\circ} \mathrm{C}$ to $70^{\circ} \mathrm{C}$. Figure 3 shows the prototype of the LoRa node with the sensors.

Finally, to carry out of experiments, we have selected an urban area with buildings of different heights. The LoRa gateway has been placed on the top of the Escuela Técnica Superior de Ingenierías Informática y de Telecomunicación (ETSIIT) of the University of Granada (Spain). The mote has travelled along the path showed in Figure 4 which also shows the level of Received Signal Strength Indicator (RSSI) of each position.

\section{Collaborative Algorithm}

This section presents operation algorithm of our proposed system as well as how it collaboratively collects the data of temperature, relative humidity and concentration of $\mathrm{CO}_{2}$.

As we explained in Figure 1, the main characteristic of our network is that it works collaboratively to collect data and to generate the most appropriate decisions to combat pollution problems in cities. As Figure 5 shows, the system is continuously receiving data from fixed nodes, mobile nodes and the own opinions from users. The received data is stored in a centralized database. The system checks if the values registered by the nodes exceed the values of thresholds. If no anomalous behavior is detected, these data are sent to a web interface where they are graphically represented on a map.

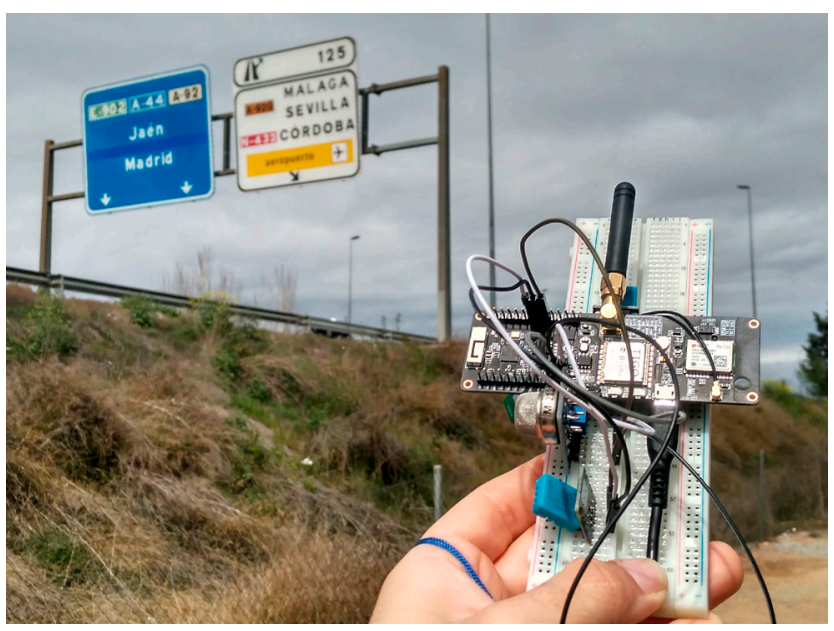

Fig. 3. Prototype of LoRa note taking measurements

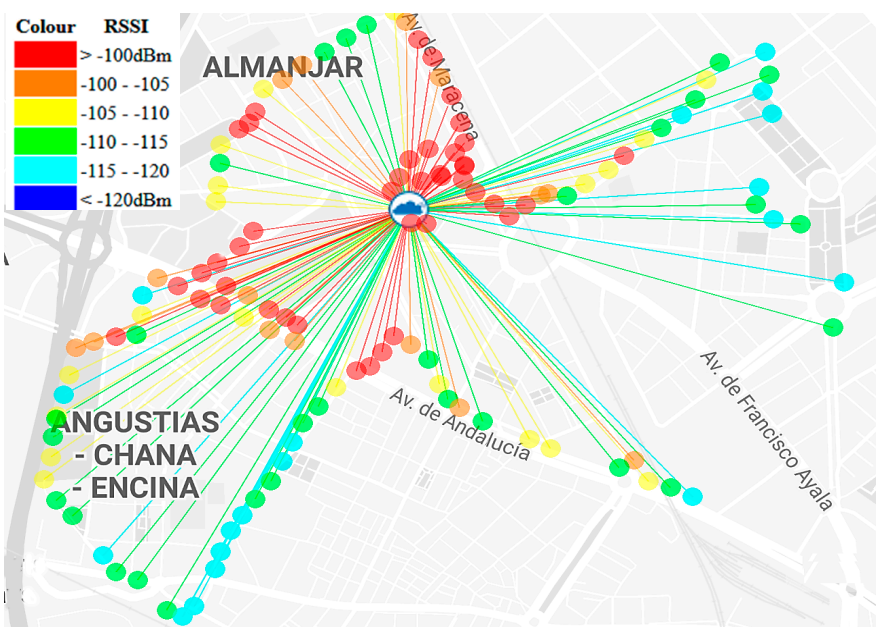

Fig. 4. Mapa de cobertura rssi

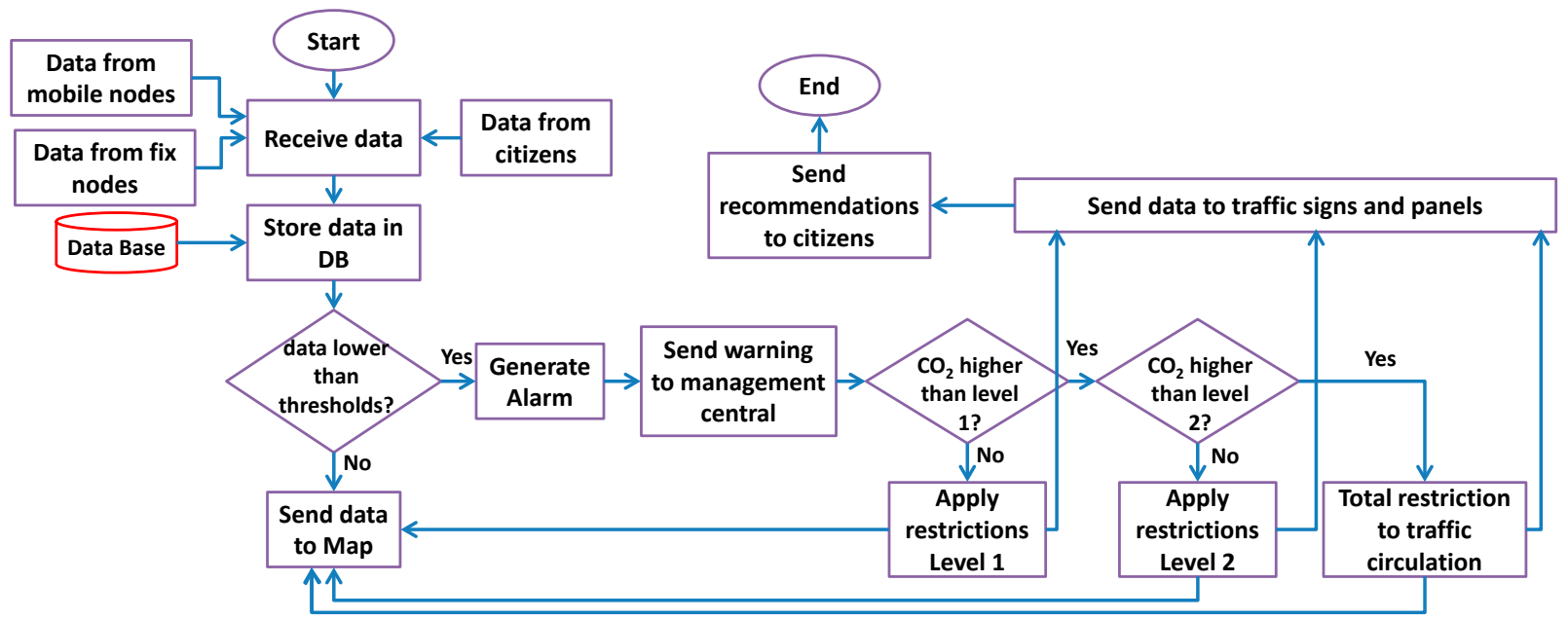

Fig. 5. Operation algoritms of collaborative LoRa-Based Sensor Network 
The data processed by the system are the GPS coordinates of the node that transmits the data, the environmental temperature, relative humidity and the $\mathrm{CO}_{2}$ concentration. If too high values are detected, the system will generate an alarm that will be sent to the local management center. After this step, the level of pollution registered in an area will be analyzed and traffic restriction policies will be applied based on the detected value. These policies are usually defined for each city. So that, a level 1 restriction may imply the reduction of the maximum speed of circulation, a restriction of level 2 may imply the reduction of the number of vehicles (considering odd and even car registration numbers) and finally higher levels of restrictions could involve the total restriction of vehicles from the affected area. After applying these restrictions, this information will be displayed in the web interface and the specific orders of those policies will be sent to the panels and traffic signals.

Finally, users will receive, through a mobile application, some recommendations about areas that should avoid to go. This information is especially useful for those people who suffer from respiratory diseases and allergies.

\section{REsults}

In order to test our system, we have used a mote as the one presented in Section III and we have followed the path explained in Section III. The tests have been divided into two different stages. The first one has collected the data form network while the second test has collected the environmental data. This section presents the obtained results of our proposal.

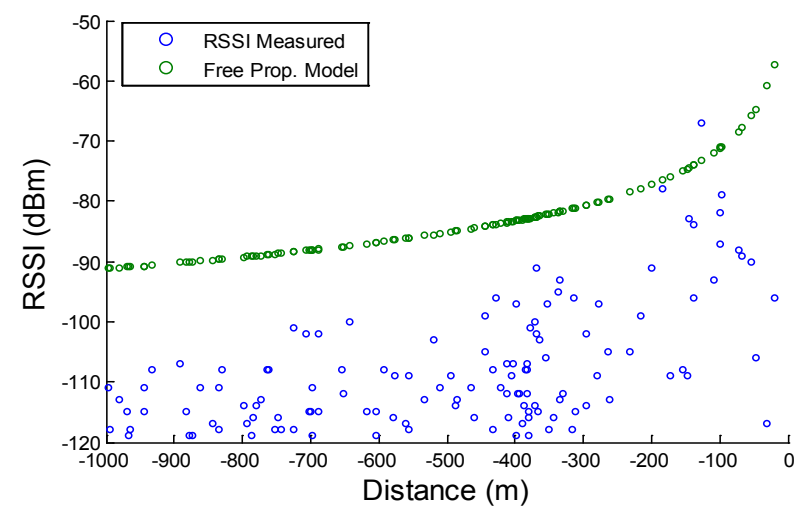

Fig. 6. Network measurement results of RSSI as a function of the distance.

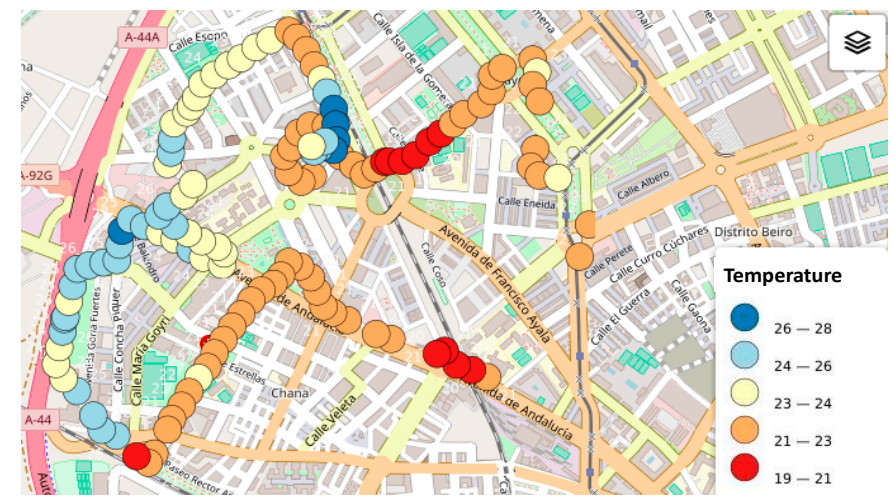

Fig. 8. Temperature measured in the test over the actual scenario

\section{A. Network measurements}

Figure 6 shows the results of RSSI obtained as a function of the distance while Figure 7 shows the results of SNR obtained as a function of the distance, taking the gateway as reference point. It should be pointed out that the nature of the selected area to perform the test have several buildings and this fact difficult the signal spread, so the presence of buildings significantly reduces the SNR and the level of the RSSI compared to the free propagation model. As we cans see, it is possible to collect data at $1000 \mathrm{~m}$ from the gateway. The average value of SNR is around $3.42 \mathrm{~dB}$ which indicate that the signal level is higher than the received noise. As a conclution, we can affirm, thar LoRa modulation robustness lets the gateway receive the packets correctly at distances higher than $1 \mathrm{~km}$.

\section{B. Measured parameters}

Figure 8, Figure 9 and Figure 10 shows the measured values of temperature, relative humidity and concentration of $\mathrm{CO}_{2}$, respectively, in the selected scenario.

As we can observe, the higher temperature values are recorded in the areas near the highway and in the access road to the University of Granada (values higher than $23{ }^{\circ} \mathrm{C}$ ), while in the rest of the areas, the temperature is maintained below 22 degrees. Directly related to these values, we observe that the relative humidity is lower in these areas. Finally we can see that the concentration of $\mathrm{CO}_{2}$ is higher in one of the main access roads to the university (with the presence of traffic lights) where depending on the time of day we can register a high volume of vehicles. We also consider that in these points we detect higher values of $\mathrm{CO} 2$ because these streets are between buildings and this fact can hinder the spread of $\mathrm{CO}_{2}$.

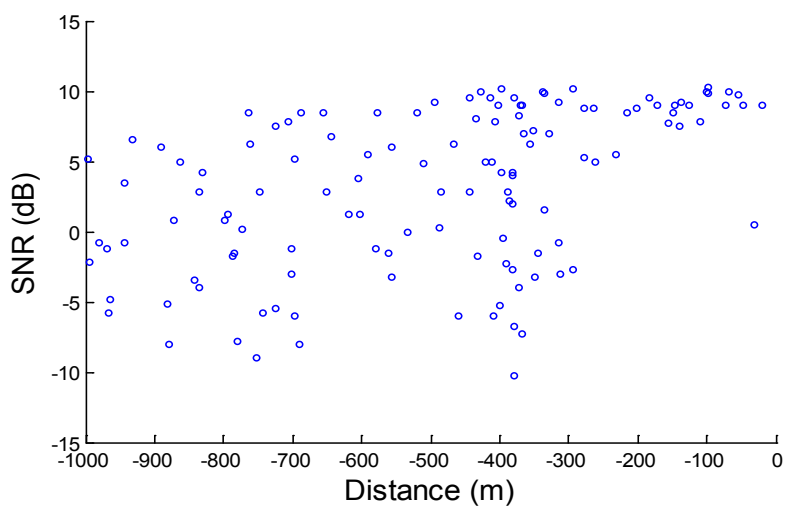

Fig. 7. Network measurement results of SNR as a function of the distance.

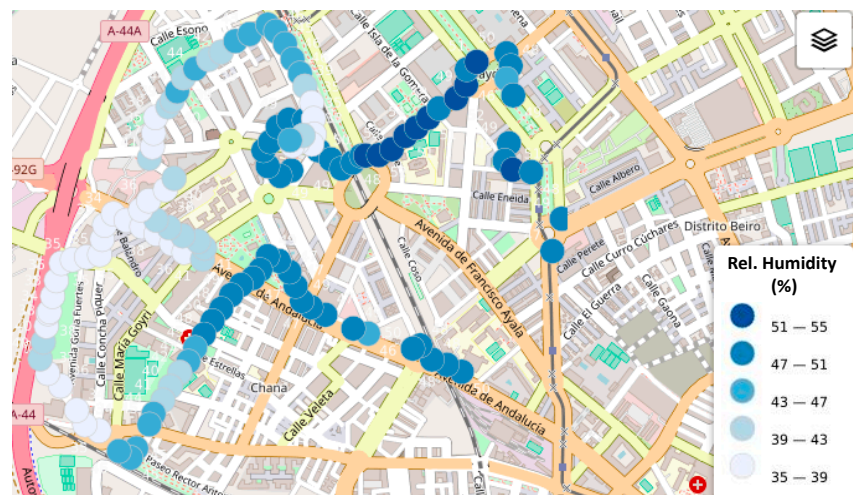

Fig. 9. Relative Humidity measured in the test over the actual scenario. 


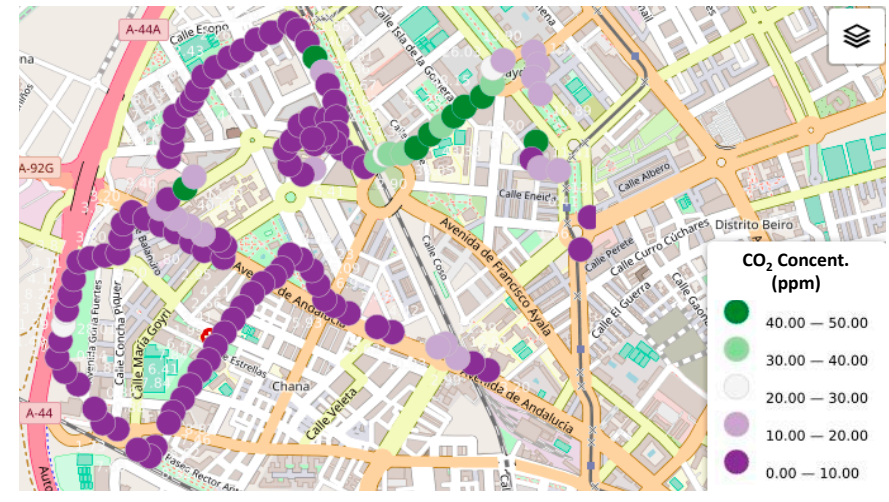

Fig. 10. $\mathrm{CO}_{2}$ (in ppm) measured in the test over the actual scenario.

\section{CONCLUSION AND FUTURE WORK}

The worrying situation of global warming is favoring the application of new technologies to create and improve the infrastructure of smart cities. The growing number of vehicles in large cities makes necessary the development of new environmental monitoring platforms to control and quantify the effect of $\mathrm{CO}_{2}$ in our environments. For this reason, in this paper, we have presented a collaborative LoRa-based sensor network to monitor the levels of pollution in smart cities. The system is composed by geo-located nodes installed in vehicles and fixed places to monitor the temperature, relative humidity and concentration of $\mathrm{CO}_{2}$ in urban environments. By using the collected data, the subjective opinions of citizens and considering the restrictions policies of the city, the system generates the required orders to panels and traffic signs which are in charge of controlling de traffic circulation. Finally, the citizens are advised to avoid walking on too polluted areas. The results shows that our system which is implemented by low-cost devices is able to easily detect de levels of $\mathrm{CO}_{2}$.

As future work, we would like to test our system in a wider area considering some additional parameters such as $\mathrm{CO}$ and volume of vehicles by image processing. Finally, we think our system could be adapted to be used in some more applications areas such as the precision horticulture [19].

\section{ACKNOWLEDGMENT}

This work has been partially supported by the European Union through the ERANETMED (Euromediterranean Cooperation through ERANET joint activities and beyond) project ERANETMED3-227 SMARTWATIR, by the "Ministerio de Economia, Industria y Competitividad", through the "Convocatoria 2016 - Proyectos I+D+I Programa Estatal De Investigación, Desarrollo e Innovación Orientada a los retos de la sociedad" (Project TEC201676795-C6-4-R), by the "Ministerio de Ciencia, Innovación y Universidades" through the "Ayudas para la adquisición de equipamiento científico-técnico, Subprograma estatal de infraestructuras de investigación y equipamiento científicotécnico (plan Estatal I+D+i 2017-2020)" (project EQC2018004988-P) and through the Research Contracts of Youth Employment of the University of Granada, through its operative program of Youth Guarantee of the Regional Government of Andalusia and the European Social Fund.

\section{REFERENCES}

[1] E. Zambom Santana, A. P. Chaves, M. A. Gerosa, F. Kon and D. S. Milojicic, "Software Platforms for Smart Cities: Concepts, Requirements, Challenges, and a Unified Reference Architecture" ACM Computing Surveys (CSUR). New York, NY, USA, vol. 50 Issue 6, Article No 78, January 2018.

[2] J. I. R. Molano, L. M. O. Bobadilla and M. P. R. Nieto, "Of cities traditional to smart cities," 2018 13th Iberian Conference on Information Systems and Technologies (CISTI), Caceres (Spain), 2018, pp. 1-6.

[3] D. Evans, "The internet of things: how the next evolution of the internet is changing everything," Cisco IBSG-White Paper, 2011.

[4] B. Hoffmann "Air pollution in cities: Urban and transport planning determinants and health in cities". in Integrating Human Health into Urban and Transport Planning. Springer, Cham, 2019. p. 425-441.

[5] K. K. Khedo, R. Perseedoss and A. Mungur, "A Wireless Sensor Network Air Pollution Monitoring System", International Journal of Wireless \& Mobile Networks (IJWMN), Vol.2, No.2, May 2010

[6] L. García, J. M. Jiménez, M. Taha, J. Lloret, "Wireless Technologies for IoT in Smart Cities," Network Protocols and Algorithms 10 (1), 23-64. 2018.

[7] A. B. Bomgni, G. B. Jagho Mdemaya, "A2CDC: Area Coverage, Connectivity and Data Collection in Wireless Sensor Networks", Network Protocols and Algorithms, Vol 10, No 4 (2018). Pp. 20-34

[8] A. J. Wixted, P. Kinnaird, H. Larijani, A. Tait, A. Ahmadinia and N. Strachan, "Evaluation of LoRa and LoRaWAN for wireless sensor networks," 2016 IEEE SENSORS, Orlando, FL, 2016, pp. 1-3.

[9] A. Augustin, J. Yi, T. Clausen and W. M. Townsley, "A Study of LoRa: Long Range \& Low Power Networks for the Internet of Things", Sensors 2016, September, 1466

[10] K. Mikhaylov, J. Petaejaejaervi and T. Haenninen, "Analysis of Capacity and Scalability of the LoRa Low Power Wide Area Network Technology," European Wireless 2016; 22th European Wireless Conference, Oulu, Finland, 2016, pp. 1-6.

[11] J. Lloret, S. Sendra, P.L. González, L. Parra "An IoT Group-Based Protocol for Smart City Interconnection." Ibero-American Congress on Information Management and Big Data. Springer, Cham, 2019.

[12] C. Lin, D. Deng, Z. Chen and K. Chen, "Key design of driving industry 4.0: joint energy-efficient deployment and scheduling in group-based industrial wireless sensor networks," in IEEE Communications Magazine, vol. 54, no. 10, pp. 46-52, October 2016.

[13] M. F. Othman and K. Shazali, "Wireless Sensor Network Applications: A Study in Environment Monitoring System", Procedia Engineering, Volume 41, 2012, Pages 1204-1210, ISSN 1877-7058.

[14] W.Y. Yi, K.M. Lo, T. Mak, K.S. Leung, Y. Leung, M.L. Meng, “A Survey of Wireless Sensor Network Based Air Pollution Monitoring Systems". Sensors 2015,15, 31392-31427.

[15] A. Kadri, E. Yaacoub, M. Mushtaha and A. Abu-Dayya, "Wireless sensor network for real-time air pollution monitoring," 2013 1st International Conference on Communications, Signal Processing, and their Applications (ICCSPA), Sharjah, 2013, pp. 1-5. doi: 10.1109/ICCSPA.2013.6487323

[16] A. Mehmood, J. Lloret, and S. Sendra. (2016) "A secure and low energy zone - based wireless sensor networks routing protocol for pollution monitoring" . Wirel. Commun. Mob. Comput., 16: 28692883. doi: $10.1002 / \mathrm{wcm} .2734$

[17] A. Mehmood, Z. Lv, J. Lloret and M. M. Umar, "ELDC: An Artificial Neural Network based Energy-Efficient and Robust Routing Scheme for Pollution Monitoring in WSNs," in IEEE Transactions on Emerging Topics in Computing. doi: 10.1109/TETC.2017.2671847

[18] C. C. Snow, D. D. Håkonsson, and B. Obel,. A smart city is a collaborative community: Lessons from smart Aarhus. California Management Review, 2016, 59(1), 92-108.

[19] C. Cambra, S. Sendra, J. Lloret and L. Garcia, "An IoT serviceoriented system for agriculture monitoring, 2017 IEEE Int. Conf. on Communications (ICC'17), Paris, France. May 21-25, 2017. pp.1-6. 